\title{
Eta Carinae: A multi-messenger source
}

\author{
Soebur Razzaque* \\ Department of Physics, University of Johannesburg \\ P.O. Box 524, Auckland Park 2006, South Africa \\ E-mail: srazzaque@uj.ac.za
}

Colliding-wind binaries have long been thought to accelerate particles in the shocked wind. Recent detection of a hard $\gamma$-ray spectrum by the Fermi-LAT from $\eta$ Carinae hints to the presence of a hadronic component dominating in the $\approx 10-300 \mathrm{GeV}$ range, presumably from protons accelerated in the shocks and interacting $(p p)$ with particles in the wind. Neutrinos are naturally produced in $p p$ interactions and emitted together with $\gamma$ rays. Detection of this multi-messenger signal can be very powerful to probe characteristics of the hadronic $\gamma$-ray component as well as particle acceleration. We show that detection of high-energy neutrinos from $\eta$ Carinae by neutrino telescopes can probe the maximum shock-accelerated proton energy in the $\gtrsim 0.1 \mathrm{PeV}$ range.

5th Annual Conference on High Energy Astrophysics in Southern Africa

4-6 October, 2017

University of the Witwatersrand (Wits), South Africa

${ }^{*}$ Speaker. 


\section{Introduction}

Gamma-ray space telescopes AGILE and Fermi-LAT have detected $\eta$ Carinae stellar binary system in the $200 \mathrm{MeV}-300 \mathrm{GeV}$ energy range [1,2]. Subsequent analyses of Fermi-LAT data collected over the full orbital period show mild orbital modulation in the $\gamma$-ray light curve and spectral hardening during the periastron passage [3, 4]. The air-Cherenkov telescope HESS has also observed $\eta$ Carinae at $\gtrsim 100 \mathrm{GeV}$ energies during the 2004-2012 period [5]. Acceleration of particles in the shocks of colliding winds of stellar binary systems and high-energy emission from therein have been discussed in literature $[6,7,8,9]$. Protons, if accelerated in the shocks, can explain the hardness of the spectrum during the periastron passage producing $\pi^{0}$ decay $\gamma$-ray flux that is dominating over the inverse-Compton flux at energies $\gtrsim 10 \mathrm{GeV}[7,4,10]$. For the hadronic component, $\pi^{0}$ is produced by $p p$ interactions of shock-accelerated protons with particles in the wind of the star, while for the leptonic component the shock-accelerated electrons Compton scatter stellar photons. The hadronic $\gamma$-ray component is accompanied by a high-energy neutrino flux originating from $\pi^{ \pm}$decays, which are coproduced with $\pi^{0}$ in the $p p$ interactions. Detection of these neutrinos can confirm hadronic origin of the $\gtrsim 10 \mathrm{GeV} \gamma$-ray component and proton acceleration in the shocks of the colliding winds of stellar binaries.

Following our lepto-hadronic model for the spectral energy distributions (SEDs) of $\eta$ Carinae [10], here we explore further characteristics of the neutrino flux models. In particular, we model neutrino fluxes based on the maximum energy that protons can be accelerated to in shocks; calculate neutrino event rates for different maximum proton energies in IceCube [16] and in the upcoming KM3NeT [17] neutrino telescopes; and compute significance of detection over the atmospheric and diffuse astrophysical neutrino backgrounds.

\section{Model details}

The winds from the binary stars $\eta$ Car-A and Car-B collide to produce shocks where particles are accelerated and emit non-thermal radiation. The electrons produce synchrotron radiation peaking at ultraviolet $(\sim 100 \mathrm{eV})$ and Compton-scattered stellar photons peaking at $\gamma$ rays $(\sim 1 \mathrm{GeV})$. The protons interact $(p p)$ with wind particles and produce $\pi^{0}$-decay $\gamma$ rays with energies $\gtrsim 100$ $\mathrm{MeV}$. The details of the radiation processes and SED modeling have been presented in [10] and the SEDs during the periastron and apastron passages are shown in Fig. 1 here. We focus on the maximum proton energy and corresponding neutrino flux modeling below.

The time scale for particle acceleration depends on the shock characteristics. In case of a perpendicular shock with magnetic field $B_{\mathrm{s}}$ the acceleration time to an energy $E$ is $t_{\mathrm{acc}}^{\perp}=8 E c / 3 \zeta e B_{\mathrm{s}} v_{w}^{2}$, where $\zeta \sim \mathscr{O}(10)$ is a multiplicative factor to the Bohm diffusion coefficient $[19,20]$. These protons interact with particles in the stellar wind with density $n_{\mathrm{H}}=\rho_{w} / m_{p}=\dot{M}_{w} / 4 \pi R^{2} v_{w} m_{p}$, where $\dot{M}_{w}$ is the mass-loss rate in wind and $v_{w}$ is the wind speed. For $\eta$ Car-A, the more massive star in the binary, $\dot{M}_{w, \mathrm{~A}}=10^{-3} M_{\odot} \mathrm{yr}^{-1}$ and $v_{w, \mathrm{~A}}=500 \mathrm{~km} \mathrm{~s}^{-1}$. The $p p$ interaction time scale for a proton over which it looses significant energy, is $t_{p p}=1 / n_{\mathrm{H}} \sigma_{p p} c$, where the average $p p$ interaction cross-section is $\sigma_{p p} \approx 30 \mathrm{mb}$. We calculate the maximum proton energy from $t_{\mathrm{acc}}^{\perp}=t_{p p}$ as [10]

$$
E_{p, \max } \approx 1\left(\frac{\zeta_{p}}{50}\right)\left(\frac{B_{\mathrm{s}, \mathrm{A}}}{5 \mathrm{G}}\right)\left(\frac{n_{\mathrm{H}, \mathrm{A}}}{2.7 \times 10^{9} \mathrm{~cm}^{-3}}\right)^{-1} \mathrm{PeV} .
$$



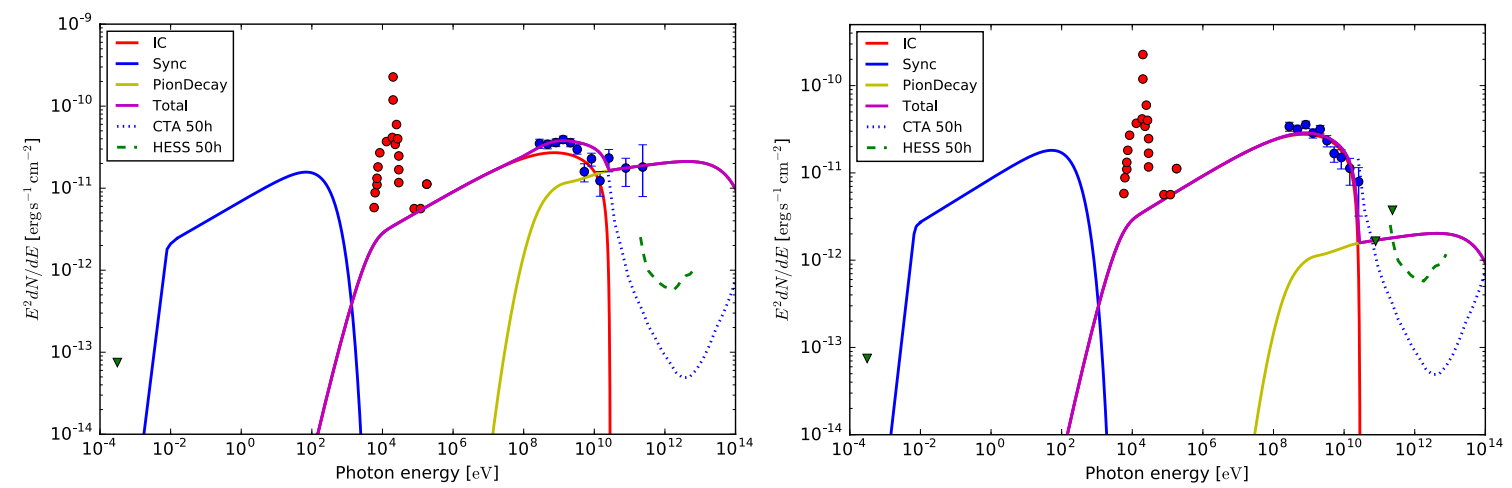

Figure 1: Spectral energy distributions (SEDs) of $\eta$ Carinae during the 500-day periastron (left panel) and 500-day apastron (right panel) passages. The radio upper limit at $3 \mathrm{~cm}$ (green downward triangle) [11] and $\mathrm{X}$-ray data (red solid circles) $[12,13,14]$ are plotted in addition to the gamma ray data (blue solid circles) $[3,4]$. The flux sensitivity curves for 50 hours observations with the CTA South and HESS are also shown [15]. Figure adapted from [10].

Fitting the $\gamma$-ray data from Fermi-LAT during the 500-day periastron passage requires $E_{p \text {,max }} \gtrsim$ $10 \mathrm{TeV}$, however the maximum cutoff energy cannot be constrained with this data. Observations with HESS or CTA in future can detect a possible cutoff in the $\gamma$-ray spectrum in the 0.1-100 TeV energy range (see Fig. 1), but absorption of $\gamma$ rays through $\gamma \gamma \rightarrow e^{ \pm}$pair production process with stellar photons $[6,9,10]$ may hinder detection of a true cutoff energy due to particle acceleration in this energy range. Neutrinos, on the hand, carry $\approx 5 \%$ of the proton energy per flavor from the $p p$ interactions and resulting $\pi^{ \pm}$decays. These neutrinos can directly probe the maximum cutoff energy in the proton spectrum while providing incontrovertible evidence of hadronic emission.

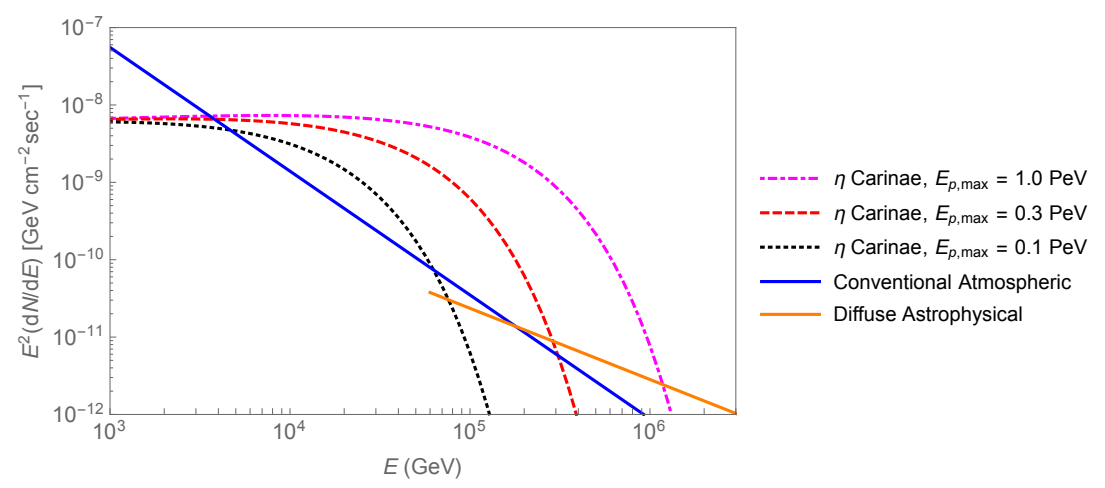

Figure 2: $\left(v_{\mu}+\bar{v}_{\mu}\right)$ fluxes from the direction of $\eta$ Carinae. The source fluxes, labeled $\eta$ Carinae, are for different cutoff energies $E_{p, \max }$ but the same spectral index in the primary proton spectrum.

In Fig. 2 we show the neutrino flux $\left(v_{\mu}+\bar{v}_{\mu}\right)$ calculations based on different values of the cutoff energy $E_{p, \max }$ in the proton spectrum $d N_{p} / d E_{p} \propto E^{-2.0} \exp \left(-E_{p} / E_{p, \max }\right)$, where the spectral index is derived from fitting $\gamma$-ray data [10,18]. The neutrino flux is $2 / 3$ of the $\gamma$-ray flux [19] per flavor, with ratio $\left(v_{e}+\bar{v}_{e}\right):\left(v_{\mu}+\bar{v}_{\mu}\right):\left(v_{\tau}+\bar{v}_{\tau}\right) \approx 1: 1: 1$. Also shown in Fig. 2 are the conventional $\left(v_{\mu}+\bar{v}_{\mu}\right)$ atmospheric flux model from [21] but extrapolated to energies $\gtrsim 63 \mathrm{TeV}$ and the diffuse astrophysical neutrino flux $E^{2}(d N / d E)=(2.46 \pm 0.8) \times 10^{-8}(E / 100 \mathrm{TeV})^{-0.92} \mathrm{GeV} \mathrm{cm}^{-2} \mathrm{~s}^{-1} \mathrm{sr}^{-1}$ 
per flavor, based on the 6-year astrophysical neutrino event data collected by IceCube [22]. The atmospheric and astrophysical fluxes in Fig. 2 have been multiplied by a solid angle $\Delta \Omega=2 \pi(1-$ $\cos \delta) \approx 10^{-3} \mathrm{sr}$, due to $\delta=1^{\circ}$ angular resolution for the track-like neutrino event signature in the detector from $v_{\mu}$ or $\bar{v}_{\mu}$ interactions.

\section{Neutrino detection}

We calculate expected neutrino event rates from the direction of $\eta$ Carinae by including the source flux averaged over $T=500$-day periastron passage and background atmospheric and diffuse astrophysical fluxes over the same period. We use publicly available effective areas $A_{\text {eff }}(E)$ of the neutrino telescopes IceCube and KM3NeT to calculate events in different energy bins $i$ as

$$
N_{i}=T \int_{E_{i, \min }}^{E_{i, \max }}\left(\frac{d N}{d E}\right) A_{\mathrm{eff}}(E) d E,
$$

where $(d N / d E)$ corresponds to the fluxes plotted in Fig. 2. Due to large angular uncertainty $\left(\delta \sim 15^{\circ}\right)$ in reconstructing the arrival directions for the cascade-type of events, created dominantly by electron or tau neutrinos and antineutrinos, we focus only on the track-like events in our calculations which are created by muon neutrinos and antineutrinos.

The declination of $\eta$ Carinae is $-59^{\circ} 41^{\prime} 04.26^{\prime \prime}$. Therefore IceCube, being located at the South pole, needs to use the high-energy starting event (HESE) selection criteria to reduce background of down-going atmospheric muons for detecting neutrinos from $\eta$ Carinae. The number of expected track-events for the 500-day periastron passage is 0.02 in the $0.1-1 \mathrm{PeV}$ neutrino energy range for the $E_{p, \max }=1 \mathrm{PeV}$ flux model. It will require integration over many periastron passages for a positive detection by IceCube.

For the upcoming Mediterranean telescope KM3NeT, the direction of $\eta$ Carinae is below the horizon. Therefore no atmospheric muon background is expected. Using parametrization of the effective areas from [17], we calculate the track-like events for KM3NeT for the 500-day periastron passage in Fig. 3. The expected angular resolution of track-like events in KM3NeT is $\lesssim 0.2^{\circ}-0.3^{\circ}$ at $E \gtrsim 10 \mathrm{TeV}$ [17]. We therefore use $\delta=0.3^{\circ}$, corresponding to $\Delta \Omega \approx 10^{-4}$ sr to calculate the conventional atmospheric and diffuse astrophysical neutrino backgrounds in Fig. 3.

We calculate significance for the detection of $\eta$ Carinae in neutrinos by KM3NeT from event distributions in Fig. 3 as $\sigma=\sqrt{T \sum_{i} S_{i}^{2} /\left(S_{i}+B_{i}\right)}$, assuming Gaussian statistics. Here $S_{i}$ and $B_{i}$ are the background events (both atmospheric and astrophysical) in the $i$-th energy bin. The detection significances during the 500-day periastron passage are $4.3 \sigma, 3.2 \sigma$ and $1.8 \sigma$; respectively for the $E_{p \text {, max }}=1 \mathrm{PeV}, 0.3 \mathrm{PeV}$ and $0.1 \mathrm{PeV}$. Since KM3NeT is expected to have full visibility throughout the year to $\eta$ Carinae [17], a lower significance detection can also be possible within a shorter time scale during the periastron passage. Furthermore, detection of tau neutrinos at these energies [23] can enhance significance further since the backgrounds are very low.

\section{Summary and outlook}

A combined leptonic and hadronic emission model can fit the SED, including 0.2-300 GeV $\gamma$ ray data from Fermi-LAT, of $\eta$ Carinae stellar binary system during its 500-day periastron passage. 


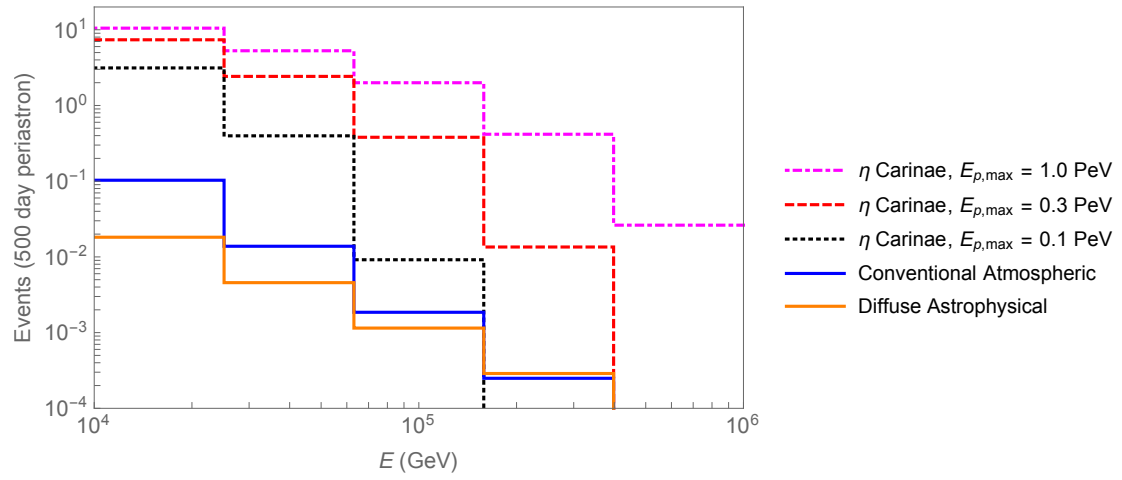

Figure 3: Expected number of track-like neutrino events in KM3NeT due to the $\left(v_{\mu}+\bar{v}_{\mu}\right)$ fluxes from the direction of $\eta$ Carinae plotted in Fig. 2. The atmospheric and astrophysical neutrino background events are calculated assuming a $0.3^{\circ}$ angular resolutions for the track-like events.

We have calculated expected neutrino fluxes based on a hadronic emission model, which dominate the SED at $\gtrsim 10 \mathrm{GeV}$. Detection of these high-energy neutrinos from $\eta$ Carinae would provide conclusive evidence of particle acceleration in colliding wind binaries and could constrain the maximum particle energy. Neutrino flux inferred from the detection can be used to calibrate the flux level of hadronic $\gamma$-ray component at $\gtrsim 100 \mathrm{GeV}$ energies, which could be attenuated in the binary environment due to $\gamma \gamma \rightarrow e^{ \pm}$pair production. We found that the upcoming neutrino telescope KM3NeT would be able to detect neutrinos from $\eta$ Carinae if the maximum shock-accelerated proton energy is $\gtrsim 0.1 \mathrm{PeV}$. Our lepto-hadronic modeling can be extended to other stellar binary systems such as WR 140, WR 147, etc. and can be used for neutrino flux prediction. Predictable neutrino emission from these systems can be stacked for enhanced detection of colliding-wind binaries by the neutrino telescopes.

\section{Acknowledgment}

This work was partially supported by grants from the University of Johannesburg and South African Gamma-ray Astronomy Programme (SA-GAMMA), funded by the Department of Science and Technology (South Africa) and the National Research Foundation (South Africa).

\section{References}

[1] M. Tavani et al. [AGILE Collaboration], "Detection of Gamma-ray Emission from the Eta-Carinae Region,” Astrophys. J. 698, L142 (2009)

[2] A. A. Abdo et al. [Fermi-LAT Collaboration], "Fermi Large Area Telescope Observation of a Gamma-ray Source at the Position of Eta Carinae,” Astrophys. J. 723, 649 (2010)

[3] K. Reitberger, A. Reimer, O. Reimer and H. Takahashi, "The first full orbit of $\eta$ Carinae seen by Fermi," Astron. Astrophys. 577 (2015) A100

[4] M. Balbo and R. Walter, "Fermi acceleration along the orbit of $\eta$ Carinae," Astron. Astrophys. 603, A111 (2017) 
[5] A. Abramowski et al. [H.E.S.S. Collaboration], "H.E.S.S. observations of the Carina nebula and its enigmatic colliding wind binary Eta Carinae," Monthly notices of the Royal Astronomical Society 424, 128 (2012)

[6] A. Reimer, M. Pohl and O. Reimer, "Non-thermal high-energy emission from colliding winds of massive stars," Astrophys. J. 644, 1118 (2006)

[7] C. Farnier, R. Walter and J.-C. Leyder, " $\eta$ Carinae: a very large hadron collider," Astron. Astrophys. 526, 57 (2011) doi:10.1051/0004-6361/201015590.

[8] W. Bednarek, J. Pabich and T. Sobczak, "Gamma-rays and neutrinos from dense environment of massive binary systems in open clusters,” Phys. Rev. D 90, no. 10, 103008 (2014)

[9] S. Ohm, V. Zabalza, J. A. Hinton and E. R. Parkin, “On the origin of $\gamma$-ray emission in $\eta$ Carina," Mon. Not. Roy. Astron. Soc. 449, no. 1, L132 (2015)

[10] N. Gupta and S. Razzaque, "Lepto-hadronic model of gamma rays from Eta Carinae and prospects for neutrino telescopes," Phys. Rev. D 96, no. 12, 123017 (2017)

[11] R. A. Duncan, S. M. White, J. Lim, et al., "Intense radio outburst from the supermassive star Eta Carinae,” Revista Mexicana de Astronomia y Astrofisica Conference Series, 2, 23 (1995)

[12] R. F. Viotti, L. A. Antonelli, C. Rossi and S. Rebecchi, "The high energy X-ray tail of $\eta$ Car revealed by BeppoSAX," Astron. Astrophys. 420, 527 (2004).

[13] J.-C. Leyder, R. Walter and G. Rauw, "Hard X-ray emission from $\eta$ Carinae," Astron. Astrophys. 477, L29 (2008).

[14] J.-C. Leyder, R. Walter and G. Rauw, "Hard X-ray identification of $\eta$ Carinae and steadiness close to periastron," Astron. Astrophys. 718, L161 (2010).

[15] B. S. Acharya et al. [Cherenkov Telescope Array Consortium], "Science with the Cherenkov Telescope Array," arXiv:1709.07997 [astro-ph.IM].

[16] A. Achterberg et al. [IceCube Collaboration], "First Year Performance of The IceCube Neutrino Telescope,” Astropart. Phys. 26, 155 (2006)

[17] S. Adrian-Martinez et al. [KM3Net Collaboration], "Letter of intent for KM3NeT 2.0," J. Phys. G 43, no. 8, 084001 (2016)

[18] C. Lunardini and S. Razzaque, "High Energy Neutrinos from the Fermi Bubbles," Phys. Rev. Lett. 108, $221102(2012)$

[19] T. K. Gaisser, “Cosmic rays and particle physics," Cambridge, UK: Univ. Pr. (1990) 279 p

[20] R. J. Protheroe, “Acceleration and interaction of ultrahigh-energy cosmic rays," In *DuVernois, M.A. (ed.): Topics in cosmic-ray astrophysics* 259-297 [astro-ph/9812055].

[21] M. Honda, T. Kajita, K. Kasahara, S. Midorikawa and T. Sanuki, "Calculation of atmospheric neutrino flux using the interaction model calibrated with atmospheric muon data," Phys. Rev. D 75, 043006 (2007)

[22] M. G. Aartsen et al. [IceCube Collaboration], "The IceCube Neutrino Observatory - Contributions to ICRC 2017 Part II: Properties of the Atmospheric and Astrophysical Neutrino Flux," arXiv:1710.01191 [astro-ph.HE].

[23] T. DeYoung, S. Razzaque and D. F. Cowen, "Astrophysical tau neutrino detection in kilometer-scale Cherenkov detectors via muonic tau decay,” Astropart. Phys. 27, 238 (2007) 